\title{
Long-term survival of elderly patients after intensive care unit admission for acute respiratory infection: a population-based, propensity score-matched cohort study
}

Antoine Guillon ${ }^{1 *}$ (D), Coralie Hermetet ${ }^{2,3}$, Kimberly A. Barker ${ }^{4,5}$, Youenn Jouan', Christophe Gaborit ${ }^{2,3}$, Stephan Ehrmann ${ }^{1,6}$, Yannick Le Manach ${ }^{7}$, Pierre-François Dequin ${ }^{1}$ and Leslie Grammatico-Guillon ${ }^{2}$

\begin{abstract}
Background: Intensive care unit (ICU) hospitalisations of elderly patients with acute respiratory infection have increased, yet the long-term effects of ICU admission among elderly individuals remain unknown. We examined differences over the 2 years after discharge in mortality, healthcare utilisation and frailty score between elderly survivors of ARI in the ICU and an elderly control population.

Methods: We used 2009-2017 data from 39 hospital discharge databases. Patients $\geq 80$ years old discharged alive from ICU hospitalisation for acute respiratory infection were propensity score-matched with controls (cataract surgery) discharged from the hospital at the same time and adjusted for age, sex and comorbidities present before hospitalisation. We reported 2-year mortality and compared healthcare utilisation and frailty scores in the 2-year periods before and after ICU hospitalisation.

Results: One thousand two hundred and twenty elderly survivors of acute respiratory infection in the ICU were discharged, and 988 were successfully matched with controls. After discharge, patients had a 10.1-fold [95\% Cl, 6.117.3] higher risk of death at 6 months and 3.6-fold [95\% Cl, 2.9-4.6] higher risk of death at 2 years compared with controls. They also had a 2-fold increase in both healthcare utilisation and frailty score in the 2 years after hospital discharge, whereas healthcare utilisation and frailty scores among controls were stable before and after hospitalisation.

Conclusions: We observed a substantially increased rate of death in the years following ICU hospitalisation for elderly patients along with elevated healthcare resource use and accelerated age-associated decline as assessed by frailty score. These findings provide data for better informed goals-of-care discussions and may help target post-ICU discharge services.
\end{abstract}

Keywords: Respiratory infection, Intensive care unit, Elderly, Epidemiology

\footnotetext{
*Correspondence: antoine.guillon@univ-tours.fr

${ }^{1}$ CHRU de Tours, Service de Médecine Intensive Réanimation, INSERM U1100, Centre d'Etude des Pathologies Respiratoires, CRICS-TriggerSEP Research

Network, Université de Tours, 2 Bd Tonnellé, F-37044 Tours Cedex 9, France

Full list of author information is available at the end of the article
}

(c) The Author(s). 2020 Open Access This article is licensed under a Creative Commons Attribution 4.0 International License, which permits use, sharing, adaptation, distribution and reproduction in any medium or format, as long as you give appropriate credit to the original author(s) and the source, provide a link to the Creative Commons licence, and indicate if changes were made. The images or other third party material in this article are included in the article's Creative Commons licence, unless indicated otherwise in a credit line to the material. If material is not included in the article's Creative Commons licence and your intended use is not permitted by statutory regulation or exceeds the permitted use, you will need to obtain permission directly from the copyright holder. To view a copy of this licence, visit http://creativecommons.org/licenses/by/4.0/ The Creative Commons Public Domain Dedication waiver (http://creativecommons.org/publicdomain/zero/1.0/) applies to the data made available in this article, unless otherwise stated in a credit line to the data. 


\section{Introduction}

The ageing of the population is a global trend of critical importance, with the number of individuals aged 80 years or older increasing the most rapidly $(3.8 \%$ increase/year) [1]. This worldwide ageing has major consequences on health systems including increasing elderly patient admissions in hospitals and intensive care units (ICUs) [2, 3]. ICU admission of elderly patients is controversial, but randomised controlled studies are difficult to design due to ethical limitations [4]. Indeed, conflicting results are present in the literature: some studies report that advanced age is itself a risk factor for ICU mortality and poor long-term quality of life due to persistent functional impairments [5-8], while other studies argue that chronological age alone should not be considered the sole criterion to preclude ICU admission [9, 10].

Most studies focusing on post-ICU effects among the elderly have considered elderly patients as a single entity. However, we feel it is questionable to discuss hospital admission policies for patients based solely on their advanced age without considering the acute disease leading to admission. Discussions of these topics should be disease-specific, and a focus on respiratory infections is of particular interest because of the strong association of these diseases with old age [11]. Consequently, we previously demonstrated a substantial increase in hospitalisations for acute respiratory infection (ARI) over 10 years (2006-2015), with a change in the ICU admission policy for elderly patients leading to an important rise in ICU resource utilisation [11]. For example, the number of nonagenarians hospitalised in ICU for ARI was 5.8-fold higher in 2015 than in 10 years earlier. However, the long-term effects of ICU admission for elderly patients with ARI have never been demonstrated.

Assessing the burden of post-ICU effects among elderly patients is particularly difficult due to the impossibility of comparing ICU-treated individuals with the most appropriate control group. Comparing elderly patients hospitalised for ARI according to whether or not they were admitted to the ICU is importantly biassed: non-ICU patients include patients that did not meet the severity criteria for ICU admission along with other cases that are sometimes severe but received only palliative care. Comparing ICU-treated patients from different age groups also does not provide useful information because of the increased risks of death and functional impairment that clearly accompany increased age.

The objective of this study was to examine the 2-year outcomes among elderly patients discharged from the hospital after being admitted to the ICU for ARI. To describe the gap in morbi-mortality between the ICU survivors and the theoretical medical condition expected at this age, we studied differences between elderly survivors of ARI and a control population representative of the physiologic effects of ageing, and matched on age, sex and preexisting comorbidities. The primary outcome was mortality, and the secondary outcomes were healthcare utilisation and frailty score over the 2 years after discharge.

\section{Methods}

\section{Data source}

A population-based cohort study was performed from January 1, 2009, to December 31, 2017, in the CentreVal de Loire region of France (2.5 million inhabitants), which is served by 39 hospitals. We used data collected from the PMSI (Programme de Médicalisation des Systèmes d'Information) national hospital discharge database. This national database is made based on the mandatory notification of each hospital stay for all French public or private hospitals. All hospitalisation information is stored in a coded summary using the International Classification of the Diseases, tenth revision (ICD-10) and the French current procedural terminology for what occurred during the hospital stay. All patients are assigned a unique identification number, allowing the same individual to be followed over time [12].

\section{Case definitions}

We defined cases of "ARI" and "cataract surgery" using an ICD-10 algorithm based on the coding resume and the French current procedure terminology coded featuring the discharge summary. ARI included diagnosis codes for acute exacerbation of chronic obstructive pulmonary disease (AECOPD) and community-acquired pneumonia (CAP) (ICD-10 diagnosis codes provided in Additional file 1). Hospitalised patients who received at least one of these ICD-10 diagnosis codes as (1) the primary diagnosis in their discharge summary or (2) the secondary diagnosis with a primary diagnosis of respiratory failure were defined as having been hospitalised with ARI. The selection of hospital stays for "cataract surgery" was performed based on the codes for cataract surgery in discharge summaries. For each patient, the following data were extracted: age, sex, primary diagnosis, comorbidities (see Additional file 1 for ICD-10 diagnosis codes used) and hospital frailty risk score at admission (according to the ICD-10 codes from the two previous years of hospital discharge summaries and measured as a continuous quantitative score from 0 to 99 [13]). The procedures performed during the ICU stay and the hospitalisation characteristics were also recorded for the ARI patients.

\section{Study population}

We included all patients aged 80 years or older who were hospitalised at least one time between January 1, 
2011, and December 31, 2013, and had one of two clinical profiles: (1) patients admitted to the ICU for ARI and discharged home alive as the population of interest and (2) patients admitted for cataract surgery and discharged home as the control population to address our expectation of spontaneous complications and death attributable to the age of this population; we matched the sex, age and comorbidities of the ARI patients with this control population selected from patients undergoing cataract surgery at the same time. We made the assumption that cataract surgery had an insignificant impact on mortality [13] and assumed that these control patients were an accurate representation of the physiological agerelated prevalence of chronic diseases and mortality.

For each patient hospitalised with ARI in the ICU and discharged alive from the hospital, a corresponding comparison patient hospitalised for cataract surgery (1:1 ratio) was selected on the basis of the nearest propensity score using the one-to-one nearest neighbour method (with a calliper of 0.002 of the standard deviation of the propensity score on the logit scale [14]) with no replacement. Matching was performed based on preexisting conditions identified over a 2-year period prior to the hospitalisation event (age, sex, frailty score, chronic heart diseases, chronic lung diseases and cancers). Hence, we used the long-term outcomes (mortality, healthcare utilisation, frailty score evolution) as proxy measures of the burden of post-ICU effects for patients 80 years of age or older who were hospitalised for ARI. After matching, the balance of covariates between the two groups was assessed using the standardised differences expressed as a percentage overall and for each covariate using the L1 measure (for which a value of 0 indicates identical distributions between groups and 1 indicates complete imbalance) and the post-matching C-statistic (for which a standardised mean difference of 0.05 or less indicates a negligible difference between the means of the two groups and a perfect balance).

Data on the propensity-matched patients with "ARI" or "Cataract" patients were then collected for the 2-year period preceding the hospitalisation (2009-2010) and for the 2year period following hospital discharge (2014-2015). Mortality was studied over a 2-year period (2014-2015). To accurately achieve this aim, the ICD-10 algorithm examined the 4-year period (2014-2017) to capture more information on the living or dead status (i.e., being alive in 2017 indicated an individual was alive at the end of 2015).

\section{Outcomes}

We furthermore studied the 2-year risk of mortality for patients 80 years or older who were discharged home alive after ICU stay for ARI compared with the expected mortality risk of this age group as determined from the controls. Mortality refers to mortality at hospital, which was defined as death during readmission during the follow-up period.

We studied healthcare utilisation in the 2-year periods preceding and following the hospital stay of interest in the matched population. A patient's care utilisation was determined by the number of days spent as an inpatient during these periods (i.e., the percentage of inpatient days per quarter). Inpatient care included outpatient visits (each one counting for 1 day of healthcare use), ambulatory care at a hospital (1 day), hospital stays (number of days spent at a hospital) and stays at rehabilitation facilities (number of days spent in the facility). Each patient acted as their own control between the two periods. We also assessed the evolution of the frailty score from the 2-year period before the hospital stay of interest through the 2-year period after this hospital stay.

\section{Ethical approval}

No nominative, sensitive or personal data on patients have been collected. Our study involves the reuse of already recorded and anonymised data. The study falls within the scope of the French Reference Methodology MR-005 according to 2016-41 law dated 26 January 2016 on the modernisation of the French health system, which require neither information nor non-opposition of the included individuals. Access to linked anonymous file in the PMSI databases was approved by the French National Commission for Data Protection and Liberties (CNIL MR-005 number 4116221019).

\section{Statistical analyses}

Continuous variables were compared using parametric or nonparametric methods, as appropriate, for paired (paired Student $t$ tests or Wilcoxon matched-pairs signed rank tests) or unpaired (Student $t$ tests or MannWhitney tests) data. Qualitative variables were also compared using parametric or nonparametric methods, as appropriate, for paired (McNemar tests or Fisher tests) or unpaired ( $X^{2}$ tests or Fisher tests) data. Kaplan-Meier curves were used to visualise the differences in survival between the ARI-ICU and control populations with log rank estimates; whether a patient was lost to follow-up, died or survived was specified every 6 months. Hazard ratios (HRs) and the 95\% CIs for primary outcomes were computed using Cox modelling of the matched population. $P$ values were 2 -tailed, and values less than .05 were considered significant. Relative risks of death between the two matched groups were assessed at 1,2 and 4 years after hospitalisation. Statistical analyses were carried out using $R$ software [15] version 3.1, and matching procedures were performed using the MatchIt package [16], version 2.4-21. Hazard ratios were determined using the survival package $[17,18]$. 


\section{Results}

\section{Matched study population}

During the inclusion period (2011-2013), 12,646 patients 80 years or older were hospitalised for ARI. Among them, 1658 patients were hospitalised in ICU and 438 (26.4\%) of those patients died during the stay. Table 1 reports the baseline characteristics and specific care support provided in ICU for the 1220 patients with ARI who were discharged alive from the hospital. During the same period, 18,921 patients were admitted for cataract surgery. The mortality during hospitalisation for cataract surgery was $0.02 \%$ (3 patients). Using the 1220 patients discharged alive from ICU hospitalisation for ARI, the matching procedure resulted in a propensitymatched cohort of 1976 patients of 80 years or older, including 988 patients hospitalised for ARI in ICU and 988 patients who underwent cataract surgery. After matching on preexisting conditions identified before the hospitalisation, there was no statistically significant difference in baseline characteristics between the patient groups (Table 2). The initial ARI cohort and the matched ARI patients are presented in Table 1.

\section{Long-term mortality}

The Kaplan-Meier curves for elderly patients discharged alive after ICU hospitalisation for ARI and for the control population showed a significant difference in mortality for these two groups (log rank test $<0.0001$, Fig. 1). Patients discharged alive after ARI in the ICU had a 10.1-fold [95\% CI, 6.1-17.3] higher risk of death at 6 months (mortality rates were 1.7\% (15/897) and $17.2 \%$ $(145 / 844)$ in matched controls and ARI patients, respectively) and a 3.6-fold [95\% CI, 2.9-4.6] higher risk of death at 2 years (mortality rates were $10.3 \%(85 / 822)$ and $37.5 \%(283 / 754)$ in matched controls and ARI patients, respectively).

\section{Healthcare utilisation}

Figure 2a reports the healthcare utilisation during the 2year periods before and after the initial hospital stay. The healthcare utilisation after ARI substantially increased in the 6 months after discharge and remained higher than the utilisation in the matched control population during the 2-year follow-up period $(P<0.0001)$. Comparatively, for the matched controls, the mean percentage of days spent in the hospital during the 2-year period after surgery slightly increased after the cataract surgery, but this increase did not reach statistical significance $(1.53 \pm 0.35 \%$ of days before the procedure vs $1.88 \pm 0.24 \%$ of days after the procedure, $p=0.07$ ). In the patients with ARI, we demonstrated a 2-fold increase in healthcare utilisation after being discharged alive from

Table 1 Baseline characteristics of elderly patients with ARI who were discharged from the hospital ICU. Patients' characteristics and specific care supports during the ICU stay are presented for both the total ARI cohort and the ARI cohort after matching was applied

\begin{tabular}{|c|c|c|}
\hline & ARI patients $(\boldsymbol{n}=1220)$ & ARI patients after matching $(\boldsymbol{n}=988)$ \\
\hline Sex ratio $(M / F)$ & 1.2 & 1.0 \\
\hline \multicolumn{3}{|l|}{ Comorbidities $(n, \%)$} \\
\hline Chronic pulmonary diseases & $274(22.5)$ & $109(11.0)$ \\
\hline Diabetes & $181(14.8)$ & $124(12.5)$ \\
\hline Obesity & $122(10.0)$ & $74(7.5)$ \\
\hline Chronic liver diseases & $14(1.2)$ & $11(1.1)$ \\
\hline Cancer & $140(11.5)$ & $91(9.2)$ \\
\hline Chronic renal diseases & $142(11.6)$ & $75(7.6)$ \\
\hline Chronic heart diseases & $530(43.4)$ & $336(34.0)$ \\
\hline Neurological diseases & $212(17.4)$ & $126(12.8)$ \\
\hline SAPS II (mean [95\% Cl]) & $38.2[37.5 ; 39.0]$ & $38.3[37.5 ; 39.1]$ \\
\hline \multicolumn{3}{|l|}{ Specific care supports } \\
\hline \multicolumn{3}{|l|}{ Mechanical ventilation } \\
\hline Invasive $(n, \%)$; duration (day, mean [95\% CI]) & $194(15.9) ; 6.5[5.6 ; 7.4]$ & $161(16.3) ; 6.6[5.6 ; 7.6]$ \\
\hline Non-invasive $(n, \%)$; duration (day, mean $[95 \% \mathrm{Cl}]$ ) & $355(29.1) ; 3.6[3.2 ; 3.9]$ & $269(27.2) ; 3.2[2.9 ; 3.6]$ \\
\hline Vasopressor $(n, \%)$ & $127(10.4)$ & $108(10.9)$ \\
\hline Renal replacement therapy $(n, \%)$ & $24(2)$ & $17(1.7)$ \\
\hline \multicolumn{3}{|l|}{ Length of stay (day) } \\
\hline In all inpatient units: mean [95\% Cl] & $16.4[15.7 ; 17.1]$ & $16.3[15.6 ; 17.1]$ \\
\hline In ICU: mean [95\% CI] & $6.3[5.9 ; 6.7]$ & $6.3[5.9 ; 6.7]$ \\
\hline
\end{tabular}


Table 2 Pre-hospitalisation patient characteristics of the matched study population. Patients discharged home after ICU hospitalisation for ARI or cataract surgery (control patients) during the same period were selected. The matching was performed based on preexisting conditions identified during the 2-year period before the hospitalisation

\begin{tabular}{llll}
\hline & Patients with ARI & Patients with cataract surgery & $\boldsymbol{p}$ value \\
\hline Number & 988 & 988 & $85.2[84.9 ; 85.4]$ \\
Age (years, mean [95\% CI]) & $85.1[84.8 ; 85.3]$ & $495(50.1)$ & 0.623 \\
Female sex (n, \%) & $494(50.0)$ & & 1.000 \\
Comorbidities (n, \%) & & $356(36.0)$ & 0.370 \\
$\quad$ Chronic heart failure & $336(34.0)$ & $105(10.6)$ & 0.828 \\
$\quad$ Chronic pulmonary diseases & $109(11.0)$ & $79(8.0)$ & 0.378 \\
$\quad$ Cancer & $91(9.2)$ & $2.78[2.50 ; 3.06]$ & 0.608 \\
Frailty score (mean [95\% Cl]) & $2.88[2.62 ; 3.15]$ & &
\end{tabular}

the hospital: the mean percentage of days spent in the hospital per quarter was $1.86 \pm 0.63 \%$ of days before the hospitalisation and $3.58 \pm 0.79 \%$ of days after the hospitalisation over a 2 -year period $(p=0.005)$. The number of days spent in a long-term care hospital drastically increased during the first 6 months post-discharge from ICU hospitalisation for ARI and then returned to the control mean estimates (estimates for the matched controls and for ARI patients during the pre-hospitalisation comparative period) (Fig. 2b).

\section{Frailty risk score}

Since frailty is potentially a determinant of the care resources needed, the evolution of hospital frailty risk score is presented in Fig. 3. We observed a 2 -fold increase in the hospital frailty risk score among the ARI patients in the 2 years post-ICU, from 2.17 [95\% CI, $1.83-2.52$ ] to 4.28 [95\% CI, 3.76-4.81] (paired analysis of 472 patients still living 2 years after being discharged from the hospital, $p<0.0001$ ); whereas the hospital frailty score of the controls slightly increased in the 2 years post-hospitalisation $+4 \%$ (from 2.52 [95\% CI, $2.22-2.83$ ] to 2.62 [95\% CI, 2.23-3.00] (paired analysis of 737 patients still living 2 years after cataract surgery, $p=0.002)$ ).

\section{Discussion}

We demonstrated that patients over 80 years old hospitalised in ICU for ARI were discharged alive from the hospital in $75 \%$ of cases. However, these patients had a 10 -fold increased risk of death 6 months posthospitalisation and 3.6-fold increased risk of death at 2 years post-hospitalisation as compared to a control population. Further, during the 2-year post-ICU period, healthcare utilisation and frailty score doubled among the ARI patient group.

We performed a propensity-matched population analysis of patients with ARI admitted in ICU compared to

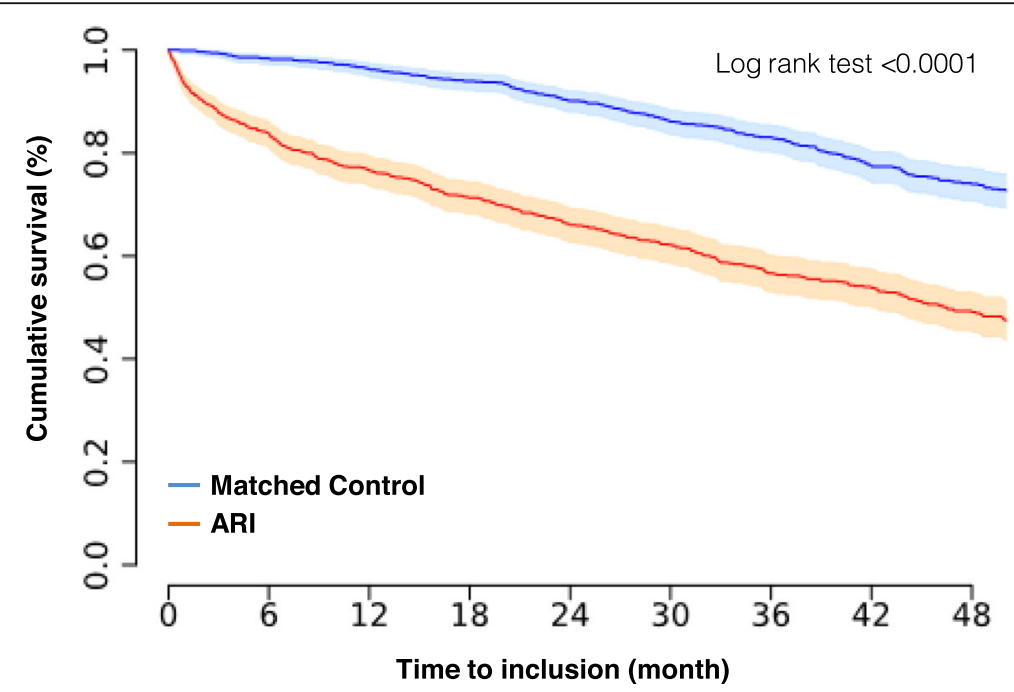

Fig. 1 Kaplan-Meier curves showing the cumulative probabilities of survival. Light colours mark the 95\% Cls of the corresponding curves (ARI, acute respiratory infection) 

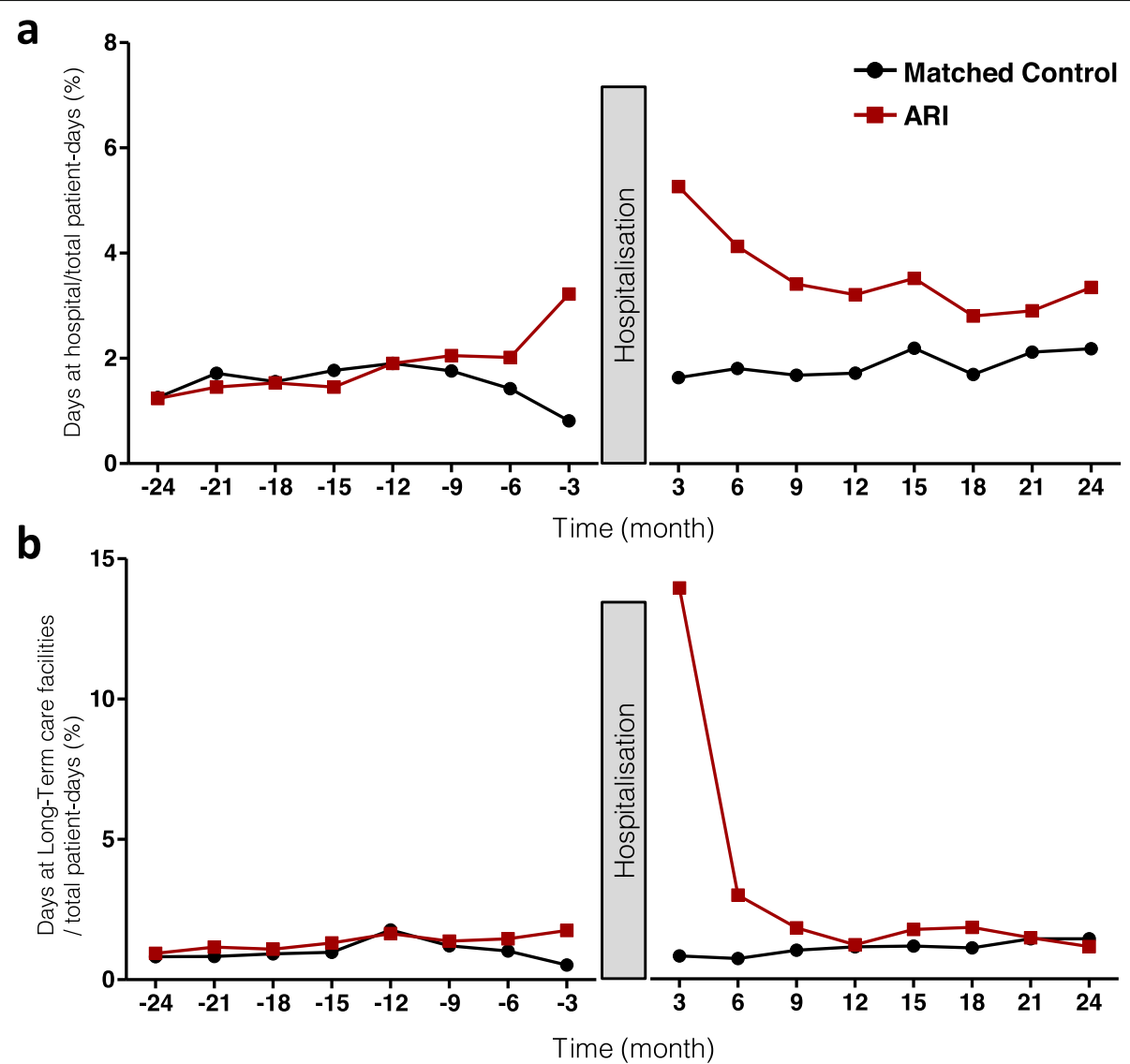

Fig. 2 Healthcare utilisation before and after initial hospital stay. The number of days spent as an inpatient in a hospital (a) or in long-term care facilities (b) during the 2-year periods before and after the initial stay (i.e., the percentage of days spent in the hospital per quarter)

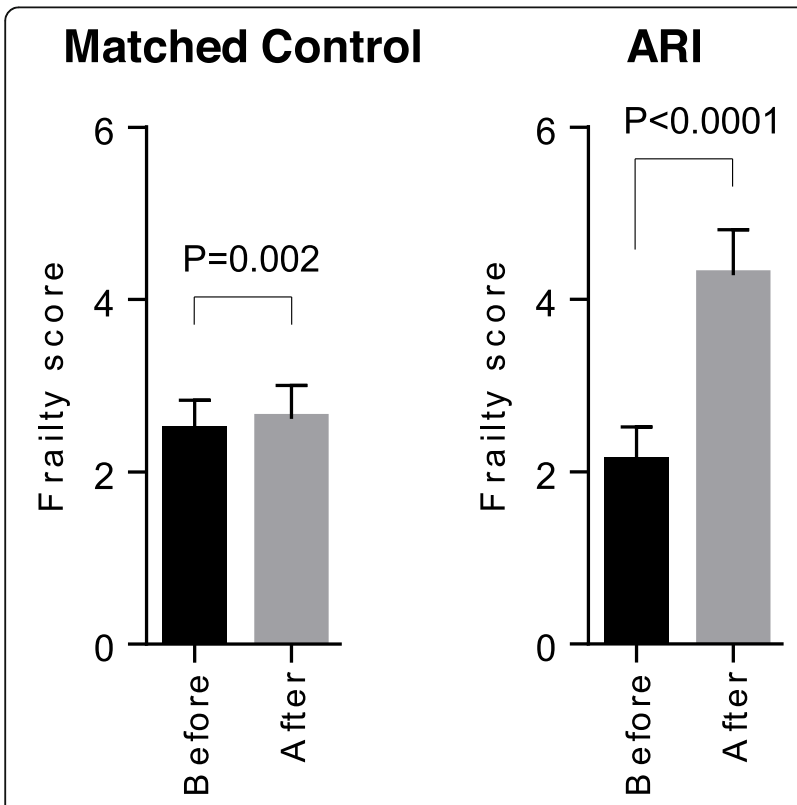

Fig. 3 Evolution of the frailty score during the 2-year periods before and after the initial hospital stay. Paired analyses performed only in patients still living 2 years after being discharged from the hospital ( $n=737$ for matched controls, $n=472$ for ARI) patients undergoing cataract surgery, which was assumed to have no effect on survival or future rehospitalisation. The insignificant mortality observed during the stays for cataract surgery combined with the comparable pre- and post-healthcare utilisation and frailty score evaluations confirmed this population as a relevant control. Importantly, matching was performed based on comorbidities that were present before the critical illness of ARI patients. As the populations were comparable in terms of age, sex and underlying diseases before the acute illness, one can attribute the excess mortality observed after hospital discharge to the severe illness and associated treatment (ARI requiring ICU hospitalisation) and not to spontaneous ageing or consequences of preexisting underlying conditions. Our study shows that an elderly patient being discharged alive from ICU is clearly not the end of the story, but rather an important turning point in life. The main findings of this study could be expressed from binary viewpoints. On one hand, as a group, a substantial proportion of elderly patients will die in the 6 months after discharge from the ICU. Moreover, the survivors will endure a critical decrease in their quality of life, as suggested by the 
worsening of the frailty score and the increase in healthcare use we observed after hospitalisation. On the other hand, on an individual level, many elderly patients surviving ICU hospitalisation will live more than 4 years after discharge (239 patients among 988). These findings highlight the need for early consideration of the management of frailty and newly developed comorbidities after ICU discharge, and more generally for management of the overall post-ICU rehabilitation process. Taking the management of hip fractures in elderly patients as an example, close cooperation between emergency physicians, anaesthesiologists, intensivists, surgeons, geriatricians and rehabilitation physicians has proven to drastically reduce the risk of death in the months after hospital discharge $[19,20]$. ARI is a severe and frequent disease among the elderly and requires the same attention. Another finding of our study is that 6 months is a more appropriate time frame to define the true ICU mortality for an elderly population, rather than the usual 30-day mortality $[19,21]$.

The present study has several strengths. First, we defined an inventive control population and matched based on preexisting conditions present before the occurrence of critical illness. By using medico-administrative databases, we were able to compare pre- and posthospitalisation trajectories and healthcare utilisation. Previous cohort studies have provided substantial knowledge on the long-term outcomes after hospital discharge [22-24]; however, prospective cohort studies are limited in the amount of information they can provide from long-term follow-up because of the lack of prehospital trajectory data [25]. Moreover, many of these longterm follow-up studies lack appropriate control groups for comparison. Consequently, there are uncertainties regarding the extent to which the morbidity and mortality observed after hospital discharge in these studies can be attributed to the critical illness and the extent to which preexisting underlying conditions contributed to such findings (which is a major limitation for elderly populations with expected comorbidities and a high mortality rate). Second, our study used a large contemporary database of 39 acute healthcare facilities in which collection of data on every hospital stay is mandatory. These data are generated based on the patient's routine care without any intervention affecting the usual methods of patient care. This study design could be assimilated as a real-life study, which contributes to the high generalisability of the results [26]. Third, in addition to survival analyses, this study assessed healthcare utilisation and frailty score evolution, which are important and relevant outcomes in an elderly population. The frailty score measures susceptibility due to the ageassociated decline in reserve and function in a wide range of physiological systems [27]. Frailty was associated with an increased risk of mortality in critically ill patients older than 80 years [28] and generally in elderly patients [29]. The doubling of the frailty score that we observed in the ICU survivors is consistent with the doubling of healthcare utilisation observed over the same time frame.

This study also has several limitations. First, the use of administrative hospital databases introduced an inherent bias that should be taken into consideration. The strengths and limitations of using healthcare databases for epidemiological purposes have already been extensively discussed [30-34]. Second, the mortality was estimated based on readmissions and mortality at hospital. Out-of-hospital deaths were not recorded, and we were thus unable to calculate the actual mortality. Based on data from the "Fin de vie en France" [End of life in France] survey, we know that dying people in France do not stay in their own homes and mostly end up in a hospital: only $14 \%$ remains at home throughout the last month of life [35]. Additionally, we expect that the rate of out-hospital deaths was similar in our experimental and control groups. Indeed, our algorithm captured the vast majority of the deaths, and we can assume that the observed increased risk of death is accurate. Third, we cannot completely exclude the possibility that there were factors affecting only one group (ARI or cataract group) that were not measured in the data available for matching. The use of two approaches (matching and pre/post analysis) demonstrated consistency and was against this latest hypothesis. However, it should be noted that only the main comorbidities, as opposed to all comorbidities, were used in matching (Table 2). The matching process also may have excluded patients with more severe illness (see Table 1). This may have led to an underestimation of the gap between morbi-mortality of elderly survivors of ARI in the ICU and theoretical values expected for this age group (represented by the control cohort). Moreover, patients were not matched on socioeconomic status and residential status (assisted living, etc.), both of which would have at least some impact on the dependency measures. Fourth, it is likely that triage based on the appropriateness of ICU admission for elderly patients was very selective. Elderly individuals are admitted to ICU only if a high likelihood of survival is expected a priori. Consequently, our results can only be extrapolated to health systems with equivalent elderly triage processes. In particular, this study is specific to the French healthcare setting and may not be generalisable. Finally, we did not include elderly patients with ARI who were admitted in the general ward as controls. Having an intermediate group of patients who survived ARI without ICU admission would have conferred additional strength to this study and given insight to any potential "dose-response" where higher illness severity potentially 
increases the long-term burden on elderly patients recovered from acute illness. However, patients were highly heterogeneous in the general ward and were comprised of those (i) not severe enough for ICU admission and (ii) meeting the criteria for ICU admission but who were too old and/or had too much comorbidity. The survivors in this heterogeneous population are difficult to characterise, because their reasons for ICU nonadmission cannot be captured by the ICD-10 algorithms. Moreover, these two populations ("elderly in general ward" and "elderly in ICU") were by definition different, because the physicians in charge of each patient initially made a call as to whether ICU admission was appropriate. Thus, trying to match these two groups by a propensity score is likely inappropriate. A prospective randomised controlled trial is probably the ideal method to answer the question of ICU benefits and the burden of post-ICU effects, but ethical concerns limit the feasibility of this approach.

\section{Conclusion}

Our data provide insight into outcomes during a 2-year follow-up of patients over 80 years old who were discharged alive from ICU after severe respiratory infection. We demonstrated an important increase in the rate of death in the years after hospital discharge along with elevated healthcare resource use and accelerated ageassociated decline as assessed by the frailty score. These findings provide data for more informed goals-of-care discussions and may help target post-ICU discharge services for these high-risk patients.

\section{Supplementary information}

Supplementary information accompanies this paper at https://doi.org/10. 1186/s13054-020-03100-4.

Additional file 1: Supplementary information on Methods. ICD-10 diagnosis codes used.

\section{Abbreviations}

ARI: Acute respiratory infection; CNIL: Commission Nationale de I'Informatique et des Libertés; CVL: Centre Val-de-Loire; HDD: Hospital discharge database; ICU: Intensive care unit; INSEE: Institut National de la Statistique et des Etudes Economiques; PMSI: Programme de Médicalisation des Systèmes d'Information; SD: Standard deviation

\section{Acknowledgements}

We thank all staffs of CVL Hospitals who contributed to the Hospital Discharge Database implementation.

\section{Authors' contributions}

AG and LGG conceived and designed the study and were involved in drafting the manuscript. $\mathrm{CH}$ and $\mathrm{CG}$ performed data retrieval. $\mathrm{CH}$ and $\mathrm{CG}$ performed the statistical analysis. YJ, KAB, YL, SE and PFD made substantial contributions to the conception of the study and interpretation of the data were involved in drafting the manuscript and made critical revisions to the discussion section. All authors read and approved the final version to be published.

\section{Funding}

The authors declare that they have no sources of funding for the research.

\section{Availability of data and materials}

The data that support the findings of this study are available from CVL Hospitals, but restrictions apply to the availability of these data and so are not publicly available. However, data are available from the authors upon reasonable request and with the permission of the institution.

\section{Ethics approval and consent to participate}

No nominative, sensitive or personal data on patients have been collected. Our study involves the reuse of already recorded and anonymised data. The study falls within the scope of the French Reference Methodology MR-005 according to 2016-41 law dated 26 January 2016 on the modernisation of the French health system, which require neither information nor nonopposition of the included individuals. Access to linked anonymous file in the PMSI databases was approved by the French National Commission for Data Protection and Liberties (CNIL MR-005 number 4116221019).

\section{Consent for publication}

Not applicable

\section{Competing interests}

Consultancies: Aerogen Ltd. (SE), La diffusion technique française (SE) and Becton Dickinson (AG). Research support: Aerogen Ltd. (SE), Fisher \& Paykel (SE) and Hamilton (SE). Travel fees: Aerogen Ltd. (SE) and Fisher \& Paykel (SE). Others authors have no competing interests to declare.

\section{Author details}

${ }^{1}$ CHRU de Tours, Service de Médecine Intensive Réanimation, INSERM U1100, Centre d'Etude des Pathologies Respiratoires, CRICS-TriggerSEP Research Network, Université de Tours, 2 Bd Tonnellé, F-37044 Tours Cedex 9, France. ${ }^{2} \mathrm{CHRU}$ de Tours, Epidémiologie des Données Cliniques (EpiDcliC), Service d'Information Médicale, d'Épidémiologie et d'Économie de la Santé, Université de Tours, Tours, France. ${ }^{3}$ EA EES, Université de Tours, Tours, France. ${ }^{4}$ Pulmonary Center, Boston University School of Medicine, Boston, MA, USA. ${ }^{5}$ Department of Microbiology, Boston University School of Medicine, Boston, MA, USA. ${ }^{6}$ CIC INSERM 1415, Tours, France. ${ }^{7}$ Departments of Anesthesia \& Clinical Epidemiology and Biostatistics, Faculty of Health Sciences, Michael DeGroote School of Medicine, McMaster University and the Perioperative Research Group, Population Health Research Institute, Hamilton, ON, Canada.

Received: 31 January 2020 Accepted: 17 June 2020

Published online: 29 June 2020

\section{References}

1. ONU. World population ageing 2013, statistical papers - United Nations (Ser. A), population and vital statistics report. New York: ONU; 2014. https://doi. org/10.18356/30d0966c-en.

2. Nielsson MS, Christiansen CF, Johansen MB, et al. Mortality in elderly ICU patients: a cohort study. Acta Anaesthesiol Scand. 2014;58:19-26. https://doi. org/10.1111/aas.12211.

3. Vosylius S, Sipylaite J, Ivaskevicius J. Determinants of outcome in elderly patients admitted to the intensive care unit. Age Ageing. 2005;34:157-62. https://doi.org/10.1093/ageing/afi037.

4. Guidet B, Leblanc $\mathrm{G}$, Simon T, et al. Effect of systematic intensive care unit triage on long-term mortality among critically ill elderly patients in France: a randomized clinical trial. JAMA. 2017:318:1450-59.

5. Ely EW, Wheeler AP, Thompson BT, et al. Recovery rate and prognosis in older persons who develop acute lung injury and the acute respiratory distress syndrome. Ann Intern Med. 2002;136:25-36.

6. Boumendil A, Maury E, Reinhard I, et al. Prognosis of patients aged 80 years and over admitted in medical intensive care unit. Intensive Care Med. 2004; 30:647-54. https://doi.org/10.1007/s00134-003-2150-z.

7. Garland A, Olafson K, Ramsey CD, et al. A population-based observational study of intensive care unit-related outcomes. With emphasis on posthospital outcomes. Ann Am Thorac Soc. 2015;12:202-8. https://doi.org/10. 1513/AnnalsATS.201405-201CME.

8. Martin-Loeches I, Guia MC, Vallecoccia MS, et al. Risk factors for mortality in elderly and very elderly critically ill patients with sepsis: a prospective, 
observational, multicenter cohort study. Ann Intensive Care. 2019;9:26. https://doi.org/10.1186/s13613-019-0495-x.

9. Andersen $\mathrm{FH}$, Flaatten $\mathrm{H}$, Klepstad $\mathrm{P}$, et al. Long-term survival and quality of life after intensive care for patients 80 years of age or older. Ann Intensive Care. 2015;5:53. https://doi.org/10.1186/s13613-015-0053-0.

10. Tabah A, Philippart F, Timsit JF, et al. Quality of life in patients aged 80 or over after ICU discharge. Crit Care. 2010;14:R2. https://doi.org/10.1186/ cc8231.

11. Laporte $L$, Hermetet $C$, Jouan $Y$, et al. Ten-year trends in intensive care admissions for respiratory infections in the elderly. Ann Intensive Care. 2018; 8:84. https://doi.org/10.1186/s13613-018-0430-6.

12. Jouan $Y$, Grammatico-Guillon L, Teixera N, et al. Healthcare trajectories before and after critical illness: population-based insight on diverse patients clusters. Ann Intensive Care. 2019;9:126. https://doi.org/10.1186/s13613-0190599-3.

13. Gilbert T, Neuburger J, Kraindler J, et al. Development and validation of a hospital frailty risk score focusing on older people in acute care settings using electronic hospital records: an observational study. Lancet. 2018;391: 1775-82. https://doi.org/10.1016/S0140-6736(18)30668-8.

14. Austin PC. The use of propensity score methods with survival or time-toevent outcomes: reporting measures of effect similar to those used in randomized experiments. Stat Med. 2014;33:1242-58. https://doi.org/10. 1002/sim.5984.

15. R Core Team (2018) R: a language and environment for statistical computing.

16. Ho D, Imai K, King G, Stuart EA. Matchlt: nonparametric preprocessing for parametric causal inference. J Stat Softw. 2011;42:1-28. https://doi.org/10. 18637/jss.v042.i08

17. Therneau TM. A package for survival analysis in S; 2015.

18. Therneau TM, Grambsch PM (2000) Modeling survival data: extending the $\{C\}$ ox model. New York: Springer.

19. Riou B, Boddaert J. The elderly patient and the ICU: where are we going where should we go? Crit Care Med. 2016;44:231-2. https://doi.org/10.1097/ CCM.0000000000001446

20. Boddaert J, Cohen-Bittan J, Khiami F, et al. Postoperative admission to a dedicated geriatric unit decreases mortality in elderly patients with hip fracture. PLoS One. 2014;9:e83795. https://doi.org/10.1371/journal.pone. 0083795.

21. Docherty AB, Anderson NH, Walsh TS, Lone NI. Equity of access to critical care among elderly patients in Scotland: a National Cohort Study. Crit Care Med. 2016;44:3-13. https://doi.org/10.1097/CCM.0000000000001377.

22. Cuthbertson BH, Roughton S, Jenkinson D, et al. Quality of life in the five years after intensive care: a cohort study. Crit Care. 2010;14:R6. https://doi. org/10.1186/cc8848

23. Herridge MS, Tansey CM, Matté A, et al. Functional disability 5 years after acute respiratory distress syndrome. N Engl J Med. 2011;364:1293-304. https://doi.org/10.1056/NEJMoa1011802

24. Lone NI, Gillies MA, Haddow C, et al. Five-year mortality and hospital costs associated with surviving intensive care. Am J Respir Crit Care Med. 2016; 194:198-208. https://doi.org/10.1164/rccm.201511-22340C.

25. Cuthbertson $\mathrm{BH}$, Wunsch $\mathrm{H}$. Long-term outcomes after critical illness. The best predictor of the future is the past. Am J Respir Crit Care Med. 2016;194: 132-4. https://doi.org/10.1164/rccm.201602-0257ED.

26. Saturni S, Bellini F, Braido F, et al. Randomized controlled trials and real life studies. Approaches and methodologies: a clinical point of view. Pulm Pharmacol Ther. 2014;27:129-38. https://doi.org/10.1016/j.pupt.2014.01.005.

27. Xue Q-L. The frailty syndrome: definition and natural history. Clin Geriatr Med. 2011;27:1-15. https://doi.org/10.1016/j.cger.2010.08.009.

28. Flaatten $\mathrm{H}$, De Lange DW, Morandi $\mathrm{A}$, et al. The impact of frailty on ICU and 30-day mortality and the level of care in very elderly patients ( $\geq 80$ years). Intensive Care Med. 2017;43:1820-8. https://doi.org/10.1007/s00134-0174940-8.

29. Brummel NE, Bell SP, Girard TD, et al. Frailty and subsequent disability and mortality among patients with critical illness. Am J Respir Crit Care Med. 2017;196:64-72. https://doi.org/10.1164/rccm.201605-09390C.

30. Jouan Y, Grammatico-Guillon L, Espitalier F, et al. Long-term outcome of severe herpes simplex encephalitis: a population-based observational study. Crit Care. 2015;19:345. https://doi.org/10.1186/s13054-015-1046-y.

31. Sunder S, Grammatico-Guillon L, Baron S, et al. Clinical and economic outcomes of infective endocarditis. Infect Dis (Lond). 2015;47:80-7. https:// doi.org/10.3109/00365548.2014.968608.
32. Grammatico-Guillon L, Baron S, Gettner $S$, et al. Bone and joint infections in hospitalized patients in France, 2008: clinical and economic outcomes. Hosp Infect. 2012;82:40-8.

33. Grammatico-Guillon L, Baron S, Gaborit C, et al. Quality assessment of hospital discharge database for routine surveillance of hip and knee arthroplastyrelated infections. Infect Control Hosp Epidemiol. 2014;35:646-51.

34. Grammatico-Guillon L, Baron $S$, Rosset $P$, et al. Surgical site infection after primary hip and knee arthroplasty: a cohort study using a hospital database. Infect Control Hosp Epidemiol. 2015;36:1198-207.

35. A majority of people would prefer to die at home, but few actually do so. In: Ined - Institut national d'études démographiques. https://www.ined.fr/ en/publications/editions/population-and-societies/die-at-home/. Accessed 12 Aug 2019.

\section{Publisher's Note}

Springer Nature remains neutral with regard to jurisdictional claims in published maps and institutional affiliations.

\section{Ready to submit your research? Choose BMC and benefit from:}

- fast, convenient online submission

- thorough peer review by experienced researchers in your field

- rapid publication on acceptance

- support for research data, including large and complex data types

- gold Open Access which fosters wider collaboration and increased citations

- maximum visibility for your research: over $100 \mathrm{M}$ website views per year

At $\mathrm{BMC}$, research is always in progress.

Learn more biomedcentral.com/submissions 